\title{
Analysis of Genetic Relationship of Native Iris species Plants using RAPD
}

\author{
Young-Hee Ahn \\ Department of Applied Plant Science, Chung-Ang University, Ansung 456-756, Korea \\ (Manuscript received 31 December, 2004: accepted 28 February, 2005)
}

This study was carried out to provide the basic data for an identifying system for Iris species distributed in Korean market from complete analysing of genetic relationship between three native Iris species and one cultivar bred from the native Iris plant. RAPD analysis of genetic relationship among 4 Irises was possible. According to the RAPD analysis, they were divided into two groups. Among 4 Irises used in this study, Iris laevigata 'Veriegata', Iris laevigata and Iris setosa were classified into the same group since they had many similarities even though the habitat of Iris laevigata in Korean peninsular is restricted mainly in the south and Iris setosa is naturally inhabited in the northern part of Kangwondo. The value for the dissimilarity index of Iris laevigata and Iris laevigata 'Veriegata' was 6.757. The value for the dissimilarity index of Iris laevigata and Iris dichotoma was 95.000 , so that they were genetically the farthest among them since the genetic relationship between two species are separated far if the value of the dissimilarity index is close to 100 .

Key Words: Iris species, Genetic relationship, RAPD, Two groups, Dissimilarity index

\section{Introduction}

Irises are monocotyledonous and perennial herbaceous plants belonging to Iridaceae ${ }^{1)}$. There are about 300 species of upright, thizomatous or bulbous, sometimes fleshy-rooted perennials found in a wide range habitats from the temperate zone to the subarctic zone in the Northern hemisphere ${ }^{2)}$.

Because flowers and leaves of Irises are very beautiful, these plants are generaily cultivated as flowering plants in order to sell their flowers, or replant in pots or flower gardens. About 10 species including Iris rossit have been found in Korea. As international exchanges of many plant species have become active recently, various Irises originally inhabited in Europe, such as Iris pseudoacorus, have been brought in Korea and distributed. Especially many garden species containing various acquired characters have been imported into Korea and planted as landscape plants. These garden species have been distributed indiscreetly over Korea

Corresponding Author: Young-Hee Ahn, Department of Applied Plant Science, Chung-Ang University, Ausung 456-756, Korea

Phone: +82-31-670-3041

E-mail: ahn3041 appost.cau.ac.kr without defining importing channel or breeding process accurately, even without analyzing what kinds of genetic characters they have been acquired or identifying which cultivars they are.

In general Irises are the vegetatively propagated plants by division or dividing, but many Iris species also propagate by seed since they seed very well. However, they become to lose their value as horticultural cultivars because nursery plants propagated by seed have undefined genetic characters from the natural crossing. For the native and cultivated Irises in Korea, therefore, an accurate survey should be done promptly in order to classify how many cultivars are grown at present and a precise identification method based on their characters should be established as well.

Iris is very difficult plant species to identify by external appearance since appearance of these plants is very simple. Because of this reason, RAPD analysis is an effective and quick method to identify genetic variation among plant species or individuals in the same species by amplifying specific DNA regions in a short time ${ }^{3)}$. Study of genetic relationship among same plant species by RAPD analysis was already reported by Ahn et $a l^{4)}$ with a Taraxacum. RAPD analysis has 
been used widely in many areas to identify genetic variation or to analyze genetic relationship among many plant species because it is a simple and quick method for the DNA polymorphism with arbitrary primers that can be easily synthesized in vitro ${ }^{5}$. Wild Iris plants used in this study were Iris dichotoma, Iris laevigata and Iris setosa that are specific Irises naturally inhabited in Daechungdo or Mt. Odae, Korea. Especially Iris laevigata is a species widely populated in Siberia, northeastern area of China and Japan including Korea. Iris laevigata 'Veriegata' is a cultivar developed for a horticultural purpose and used internationally for greening in damp area. In this study, therefore, analysis of genetic relationship among 4 Irises that have known distributed area, character and breeding process was conducted by RAPD. This study was carried out to provide the basic data for an identifying system for Iris species distributed in Korean market from complete analysing of genetic relationship between three native Iris species and one cultivar bred from the native Iris plant.

\section{Materials and Methods}

Our study was carried out from August 2002 to June 2004. Four Iris plants used in this study were listed in Table 1. The samples of Iris setosa and Iris laevigata were collected in Mt. Odae and Mt. Chiri accordingly. Iris dichotoma and Iris laevigata 'Veriegata' were collected at Pyungkang Botanical Garden located in Pochungun, Kyungkido. After keeping collected fresh leaves of each species at $4^{\circ} \mathrm{C}$ for $72 \mathrm{~h}$ to remove all carbohydrates, they were frozen by liquid nitrogen and stored in $-72^{\circ} \mathrm{C}$ deep freezer for RAPD analysis.

Primers used in PCR were the random primers in the primer kit supplied by Operon Technologies Inc.(Alameda, CA). Nucleotide sequences and GC content of each primer were shown in Table 2. PCR conditions for analysis of genetic variation between Iris plants using the random primers are as follows; Mixture of $50 \mathrm{ng}$ of each genomic DNA, 2.5 $\mu$ of

Table 1. Morphological studies of three native Iris species and one cultivar

\begin{tabular}{|c|c|c|c|c|c|c|}
\hline \multirow{2}{*}{ Scientific name } & \multirow{2}{*}{$\begin{array}{l}\text { Plant height } \\
(\mathrm{cm})\end{array}$} & \multicolumn{2}{|c|}{ Flower } & \multicolumn{3}{|c|}{ Leaf } \\
\hline & & Blooming period & Color & Length $(\mathrm{cm})$ & Width $(\mathrm{cm})$ & Color \\
\hline Iris laevigata & $50-90$ & May - June & Pansy purple & $50-90$ & $1-3$ & Green \\
\hline $\begin{array}{l}\text { Iris laevigata } \\
\text { 'Variegata' }\end{array}$ & about 70 & May - June & Pansy purple & $50-80$ & $1-3$ & $\begin{array}{c}\text { White variegated } \\
\text { plant }\end{array}$ \\
\hline Iris dichotoma & about 80 & June - July & Blue purple & $30-50$ & $1-2$ & Green \\
\hline Iris setosa & about 70 & Aug. - Sep. & Blue or purple & $20-30$ & $2-2.5$ & Green \\
\hline
\end{tabular}

Table 2. List of arbitrary 10 -mer primers used in the RAPD study

\begin{tabular}{|c|c|c|c|}
\hline \multicolumn{2}{|c|}{ Operon Primer No. } & Primer Sequence & GC Content $(\%)$ \\
\hline \multirow{2}{*}{ OPA } & 5 & 5'-AGGGGTCTTG-3 & 60 \\
\hline & 6 & 5'-GGTCCCTGAC-3 & 70 \\
\hline OPB & 1 & 5'-GTTTCGCTCC-3 & 60 \\
\hline OPC & 2 & 5'-GTGAGGCGTC-3 & 70 \\
\hline OPD & 2 & 5'-GGACCCAACC-3 & 70 \\
\hline OPF & 1 & 5'-ACGGATCCTG-3 & 60 \\
\hline OPK & 1 & 5'-CATTCGAGCC-3 & 60 \\
\hline \multirow{2}{*}{$\mathrm{OPO}$} & 2 & 5'-ACGTAGCGTC-3 & 60 \\
\hline & 3 & 5'-CTGTTGCTAC-3 & 50 \\
\hline OPS & 1 & 5'-CTACTGCGCT-3 & 60 \\
\hline \multirow{2}{*}{ OPAW } & 1 & 5'-ACCTAGGGGA-3 & 60 \\
\hline & 5 & 5'-CTGCTTCGAG-3 & 60 \\
\hline
\end{tabular}


$10 \mathrm{X}$ reaction buffer, $2 \mu \ell$ of $2 \mathrm{mM} \mathrm{MgCl}_{2}, 2 \mu l$ of $2 \mathrm{mM}$ $\mathrm{dNTPs}$, 20ng of each random primer and lunit of Taq polymerase were made and $\mathrm{dH}_{2} \mathrm{O}$ was added to make the final volume of $25 \mu \ell$. After $5 \mathrm{~min}$ of predenaturation at $95^{\circ} \mathrm{C}$, amplification reactions were carried out on the Primus 96-Plus (MWG-Biotech Inc., Germany) subjected to 55 cycles of PCR as follows; $95^{\circ} \mathrm{C}, 1 \mathrm{~min}$ denaturation; $35^{\circ} \mathrm{C}, 2 \mathrm{~min}$ annealing; $72^{\circ} \mathrm{C}, 2 \mathrm{~min}$ extension $^{6)}$.

Amplification products were analysed by electrophoresis on $1.5 \%$ of agarose gel containing $0.1 \mathrm{ug} / \mathrm{ml}$ of ethydium bromide and visualized by UV transilluminator. The DNA fingerprints were photographed with Polaroid print film. Molecular weight of each amplified DNA fragment was determined by lambda-Hind III standard marker. Based on the bands recorded as present or absent, a binary matrix was obtained. The binary matrix was transformed into a similarity matrix according to the Nei and Li's method ${ }^{7)}$ using Treecon ${ }^{8)}$. From this matrix, phenogram was obtained by cluster analysis, UPGMA method (Unweighted pair-group method with arithmatic average), and a bootstrap analysis ${ }^{9}$ was performed on it as the percent of 100 replication.

\section{Results and Discussion}

Morphological character of three native Iris, Iris setosa, Iris dichotoma and Iris laevigata, and one cultivar, Iris laevigata 'Veriegata' was examined. Flower color of Iris dichotoma is pink or violet, but flower color of Iris setosa is dark purple. Flower color of Iris laevigata is very similar to Iris setosa, but it is little light. Flower shape and color of Iris laevigata 'Veriegata' were similar to Iris laevigata, but its color is relatively light. In the central region of Korea, blooming period of Iris laevigata is the earliest time, May through June, and Iris laevigata 'Veriegata' is May through July. Blooming period of Iris dichotoma is August through September, which is the latest time among them. Leaves' shape of Iris laevigata and Iris laevigata 'Veriegata' is $40-60 \mathrm{~cm}$ that is longer than others and Iris setosa is $30-50 \mathrm{~cm}$. But, leaves' shape of Iris dichotoma is $20-30 \mathrm{~cm}$, which is the shortest leaves among them. Since Iris laevigata 'Veriegata' is a horticultural cultivar developed from Iris laevigata, they have similar morphological characteristics and white striped patterns on their leaves ${ }^{1)}$.

To analysis genetic relationship among four Irises, primer screening using 100 10mer-arbitrary primers supplied by Operon Technologies Inc.(Alameda, CA) was conducted to find primers that showed in polymorphism. A total of 41 polymorphic bands were generated using 30 primers selected by primer screening(Fig. 1). From this data, RAPD analysis of genetic relationship among 4 Irises was possible. According to the RAPD analysis, they were divided into two groups. The result showed that there was not different in the genetic variation between Iris laevigata 'Veriegata', a horticultural cultivar developed for the white striped patterns on their leaves as an ornamental value from Iris laevigata, and its original species, Iris laevigata. From comparing to these two plants during cultivating, it was easy to know that plant's height,

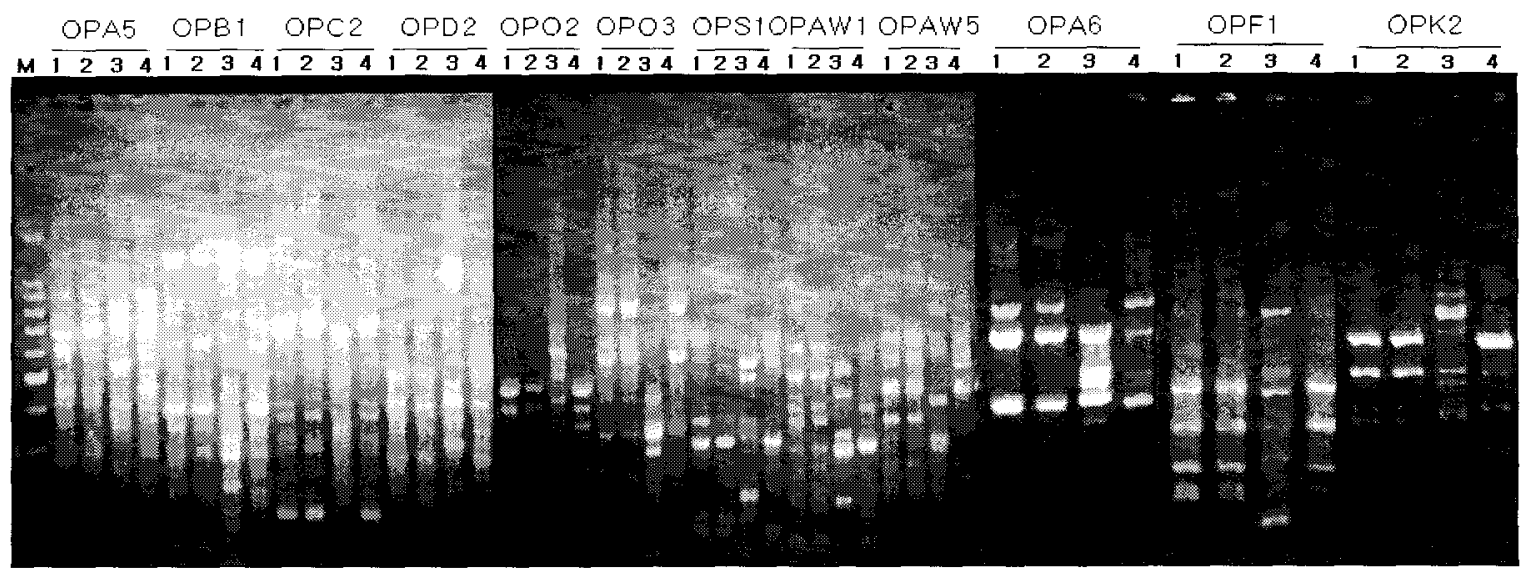

Fig. 1. RAPD band profiles of 3 lris native soecies and one cultivar separated on the $1.5 \%$ agarose gel. M : DNA size marker( $($ Hind III).

1: Iris laebigata, 2: Iris laevigata 'Variegata', 3: Iris dichotoma, 4: Iris setosa 

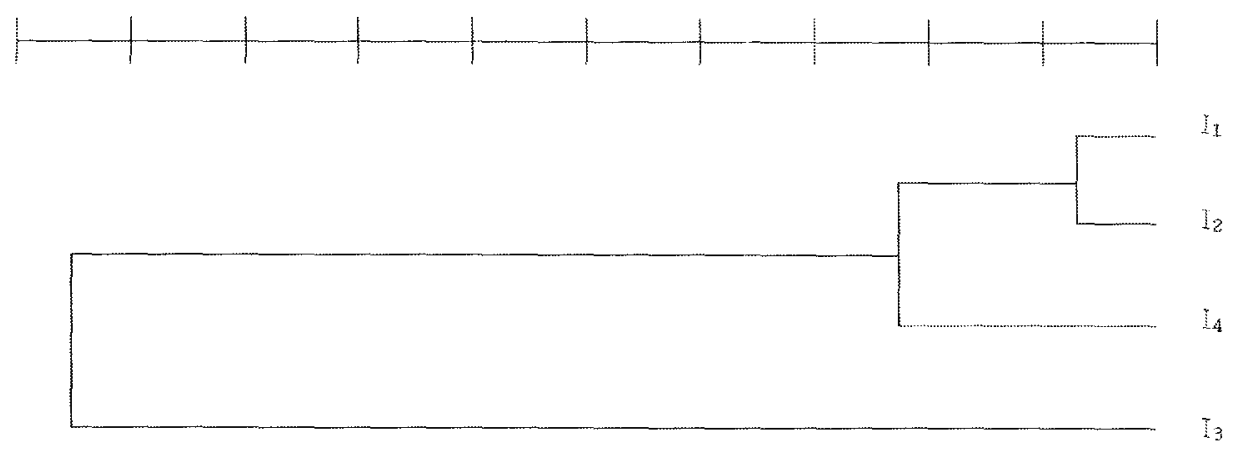

Fig. 2. Phenogram generated form Nie and Li's coefficient demonstrating the relationship between 3 native Iris species and one cultivar based on RAPD. Dissimilarity index indicates the genetic distance.

$\mathrm{I}_{1}$ : Iris laevigata, $\mathrm{I}_{2}$ : Iris laevigata 'Variegata', $\mathrm{I}_{3}:$ Iris dichotoma, $\mathrm{I}_{4}:$ Iris setosa

blooming period and flower colors were very similar each other except leaves' color and the white striped patterns on their leaves. Among 4 Irises used in this study, Iris laevigata 'Veriegata', Iris laevigata and Iris setosa were classified into the same group since they had many similarities even though the habitat of Iris laevigata in Korean peninsular is restricted mainly in the south and Iris setosa is naturally inhabited in the northern part of Kangwondo(Fig. 2). However, the morphological characteristics of flowers of Iris laevigata and Iris setosa were similar in appearance. Because habitat of Iris dichotoma classified into another group is restricted in Daechungdo and Baekryungdo located in the western sea of Korea and isolated from other species, it has several specific regional characteristics. Since flower's shape and color and leave's shape of Iris dichotoma were completely different from 3 other species, the RAPD analysis of 4 Irises showed the similar result that expected.

The dissimilarity index for 4 Irises was determined (Table 3). The value for the dissimilarity index of Iris laevigata and Iris laevigata 'Veriegata' was 6.757. Because the similarity value between each species was determined by the dissimilarity index, Iris laevigata and Iris laevigata 'Veriegata' were genetically closed each other. The value for the dissimilarity index of Iris laevigata and Iris dichotoma was 95.000 , so that they were genetically the farthest among them since the genetic relationship between two species are separated far if the value of the dissimilarity index is close to $100^{10)}$.
Table 3. Dissimilarity index (matrix) between 3 native Iris species and one cultivar

\begin{tabular}{c|rrrr}
\hline & \multicolumn{1}{|c}{$\mathrm{I}_{1}$} & \multicolumn{1}{c}{$\mathrm{I}_{2}$} & $\mathrm{I}_{3}$ & \multicolumn{1}{c}{$\mathrm{I}_{4}$} \\
\hline $\mathrm{I}_{1}$ & 0.000 & 6.757 & 95.000 & 20.000 \\
$\mathrm{I}_{2}$ & 6.757 & 0.000 & 94.937 & 25.874 \\
$\mathrm{I}_{3}$ & 95.000 & 94.937 & 0.000 & 93.548 \\
$\mathrm{I}_{4}$ & 20.000 & 25.874 & 93.548 & 0.000 \\
\hline
\end{tabular}

$\mathrm{I}_{1}:$ Iris laevigata, $\mathrm{I}_{2}:$ Iris laevigata 'Variegata', $\mathrm{I}_{3}:$ Iris dichotoma, $\mathrm{I}_{4}:$ Iris setosa

\section{Acknowledgements}

This work was conducted based on research funding from Chung-Ang University.

\section{References}

1) Lee, T. B., 1982, Illustrated flora of Korea. Hyangmoonsa publishing Co., Seoul, 638pp.

2) Hotta, M., 1989, Useful plants of the world, Heibon sh a LTD., Tokyo, 525-527pp.

3) Rani, V. A., K. Parida and S. N. Raina, 1995, Randomly amplified polymorphic DNA(RAPD) markers for genetic analysis in micropropagated plants Populus deltoides, Plant Cell Reports, 14, 459-462.

4) Ahn, Y. H., D. S. Park and K. Y. Chung, 2003, Analysis of genetic relationship among native Taraxacum and naturalized Taraxacum species using RAPD, The Korean Journal of Environment and Ecology, 17, 169-176.

5) Benner, M. S., M. D. Braunstein and M. U. Weisberg, 1995, Detection of DNA polymorphisms within the genus Cattleya (Orchidaceae), Plant Mor. Biol. Rep., 13, 147-155. 
6) Chalmer, K. J., R. Wauugh, J. K. Sprent, A. J. Simons and W. Powell, 1992, Detection of genetic variation between and within populations of Gliricidia sepium and $G$. maculata using RAPD markers, Heredity, 69, 465-472.

7) Nei, M. and W. H. Li, 1979, Mathematical model for studying genetic variation in terms of restriction endonucleases, Proc. Natl. Acad. Sci., 76, 52695273.
8) Sneath, P. H. A. and R. R. Sokal, 1973, Numerical Taxonomy, W. H. Freeman, 341-347pp.

9) Van De Peer, Y. and R. De Watcher, 1993, Treecon: A software package for the construction and drawing of evolutionary trees, Comput. Applic. Biosci., 9, 177-182.

10) Felsenstein, J., 1985, Confidence limits on phylogenies: an approach using the bootstrap, Evolution, 39, 738-791. 